\title{
Comunicación
}

\section{EVALUACIÓN DEL ESTABLECIMIENTO DEL HÍBRIDO Brachiaria ruziziensis x Brachiaria brizantha EN CONDICIONES DE TRÓPICO HÚMEDO}

\author{
ESTABLISHMENT OF THE BRACHIARIA RUZIZIENSIS X BRACHIARIA BRIZANTHA \\ Hybrid Under Humid Tropical Conditions
}

Miguel Ara G. ${ }^{1,2}$ y César Reyes A. ${ }^{1}$

\section{Abstract}

\begin{abstract}
The hybrid Brachiaria ruziziensis $\mathrm{x}$ Brachiaria brizantha (pasto Mulato) has been released as a highly productive tropical forage. This new option is being tested at Pucallpa in a field trial under a combination of NP fertilizer rates $(0,75 \mathrm{y} 150 \mathrm{~kg} / \mathrm{ha} /$ year of $\mathrm{N}$ and 0 , 25 y $50 \mathrm{~kg} / \mathrm{ha} /$ year of $\mathrm{P}$ ). Establishment evaluations, resulting from measuring seedling emergence and biomass cover at 3, 6, 9, and 12 weeks after planting, showed Mulato as a forage with an excellent establishment potential, particularly with high seedling emergence and coverage rate, and apparently tolerant to $\mathrm{N}$ deficiency, as compared with Brachiaria brizantha.
\end{abstract}

Key words: forages, Mulato, Brachiaria brizantha, establishment, Pucallpa

Las especies del género Brachiaria, especialmente $B$. decumbens, son las gramíneas forrajeras tropicales de más amplia distribución. En América Latina existen más de siete millones de hectáreas sembradas con B. decumbens, y en el Perú, desde su introducción por el Centro de Investigación IVITA en los años '70, es prácticamente la gramínea de elección en el trópico húmedo por debajo de 1,000 msnsm.

La alta productividad de biomasa y la tolerancia a suelos ácidos e infértiles han conferido al $B$. decumbens un rol preponderante en los sistemas de producción animal. Sin embargo, esta gramínea tiene problemas de calidad nutritiva variable $\mathrm{y}$, principalmente, susceptibilidad al ataque de salivazo (Homóptera: Cercopidae). Las estrategias para negociar estas limitaciones han estado dirigidas a la búsqueda de nuevo germoplasma, tanto a través de la selección in situ, como a través del mejoramiento genético. La selección específica ha producido opciones como $B$. dictyoneura y $B$. brizantha, que tienen mejores niveles de tolerancia y resistencia al salivazo que la $B$. decumbens, pero presentan una menor productividad y aceptabilidad por el ganado. Por otro lado, el Centro Internacional de Agri-

\footnotetext{
${ }^{1}$ Instituto Veterinario de Investigaciones Tropicales y de Altura, IVITA-Pucallpa, Facultad de Medicina Veterinaria, Universidad Nacional Mayor de San Marcos, Lima

${ }^{2}$ E-mail: marag@unmsm.edu.pe
} 
cultura Tropical, luego de vencer las dificultades de poliploidía y apomixis inherentes al género Brachiaria, ha generado uno de los primeros híbridos, la accesión CIAT 36061 B. ruziziensis $\mathrm{x}$ B. brizantha, con características promisorias de productividad y tolerancia a salivazo (CIAT, 2002). Esta accesión fue lanzada por primera vez en México como «pasto Mulato».

La literatura de lanzamiento de Mulato (Grupo Papalotla, 2003) lo describe como una gramínea excepcionalmente superior a $B$. decumbens y $B$. brizantha, especialmente en términos de capacidad de establecimiento, productividad de biomasa y producción de leche y carne. Sin embargo, existen dudas sobre su comportamiento en suelos del trópico peruano, debido a que uno de sus progenitores, B. ruziziensis, no es tolerante a suelos ácidos e infértiles. Esto justifica una evaluación agronómica de Mulato, especialmente al establecimiento en condiciones de trópico húmedo peruano y frente a $B$. brizantha, una de las especies forrajeras más recomendadas para la selva central.

En la Estación Experimental de Pucallpa del Centro de Investigación IVITA se instalaron 54 parcelas de $2 \times 4 \mathrm{~m}$. Se evaluaron 18 tratamientos, consistentes en una combinación factorial de tres niveles de fertilización con $\mathrm{N}(0,75$ y $150 \mathrm{~kg} / \mathrm{ha} / \mathrm{año})$ por tres niveles con $\mathrm{P}(0,25$ y $50 \mathrm{~kg} / \mathrm{ha} / \mathrm{año})$ para dos forrajes: Mulato y $B$. brizantha, que fueron distribuidos en cada uno de tres bloques espaciales en el área experimental descrita.

El Mulato (semilla experimental donada por IICA-CIAT) y la $B$. brizantha fueron sembrados en las parcelas experimentales en noviembre de 2004, a un distanciamento de $0.50 \mathrm{~m}$ entre hileras y usando las densidades recomendadas por especie: $6 \mathrm{~kg} / \mathrm{ha}$ para Mulato y $3 \mathrm{~kg} / \mathrm{ha}$ para $B$. brizantha.

El establecimiento se evaluó entre los 21 y 84 días después de la siembra. Para ello, se determinó el número de plántulas brotadas y se estimó la cobertura de la biomasa de los nueve tratamientos de fertilización y los dos forrajes experimentales a 3, 6, 9 y 12 semanas después de la siembra, usando un marco de $1 \mathrm{~m}^{2}$ (Toledo y Schulze-Kraft, 1982).

El efecto de la especie y la fertilización sobre las variables de establecimiento fue estimado mediante Análisis de Variancia correspondiente a un diseño de Bloques Completos al Azar con tres repeticiones, en arreglo de Parcelas Divididas con los nueve tratamientos de fertilización en parcelas principales y las especies en subparcelas. Se hicieron análisis estadísticos independientes para cada una de las evaluaciones $(3,6,9$ y 12 semanas).

El número de plántulas brotadas de $\mathrm{Mu}-$ lato a las tres semanas de la siembra $\left(25 / \mathrm{m}^{2}\right)$ fue significativamente superior $(\mathrm{p}=0.011)$ al de B. brizantha $\left(21 / \mathrm{m}^{2}\right)$. Esta diferencia se mantuvo en las cuatro evaluaciones y no pareció ser afectada por los niveles de $\mathrm{N}$ o de $\mathrm{P}$, ni por la combinación de ambos. En términos de capacidad de cobertura, Mulato fue superior $(\mathrm{p}=0.0001)$ a $B$. brizantha, independientemente del nivel de fertilizacion (p? 0.06), en las cuatro evaluaciones (Fig. 1).

Por otro lado, en promedio de especies $\mathrm{y}$ niveles de $\mathrm{P}$, la fertilización con $\mathrm{N}$ incrementó significativamente la capacidad de cobertura a las $6(\mathrm{p}=0.033)$ y a las 9 semanas ( $p=0.006)$. En cambio, el efecto de la fertilización con $\mathrm{P}$ fue mucho más marcado (Fig. 1), ya que se incrementó significativamente $(\mathrm{p}=0.0001)$ en promedio de especies y de niveles de $\mathrm{N}$ la capacidad de cobertura a lo largo de todas las evaluaciones.

Las tendencias de la Fig. 1 muestran que, a las densidades de siembra recomendadas, Mulato posee una alta velocidad de cobertura, la cual refleja sus características de rápida acumulación de materia seca a partir de la cuarta semana de crecimiento (Pérez et al., 2004) Dos componentes de esta característica son notables: por un lado, a las 9 semanas de la siembra, para las combinacio- 


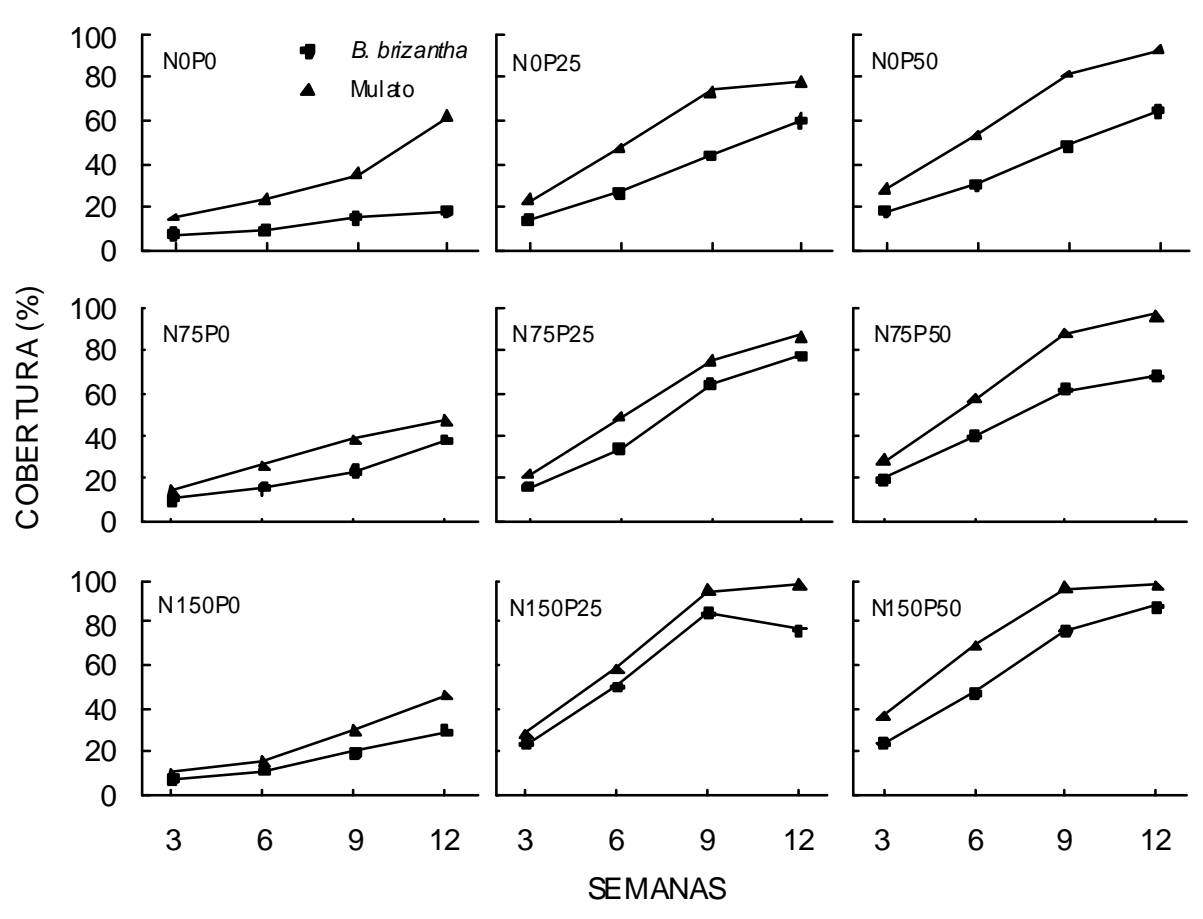

Figura 1. Evolución de la cobertura de Mulato y Brachiaria brizantha entre 3 y 12 semanas después de la siembra bajo nueve combinaciones de niveles de fertilización $(\mathrm{kg} / \mathrm{ha} /$ año) de N y P. Pucallpa, Perú. 2005.

nes de fertilización N150 P25 y N150 P50, y a las 12 semanas para la combinación N75 P50, Mulato llegó a cubrir prácticamente el $100 \%$ de la superficie, lo que no sucede en ausencia de fertilización, donde Mulato sólo alcanza una cobertura de $60 \%$ a las 12 semanas. Por otro lado, la fertilización con $\mathrm{N}$ no parece ser crítica para la velocidad de cobertura de Mulato ya que, a las 12 semanas, la cobertura alcanzada con la combinación N0 P50 no fue estadísticamente diferente a la obtenida con la combinación N150 P50. Esta característica sería una ventaja para el establecimiento de Mulato asociado con leguminosas, donde no se fertiliza con $\mathrm{N}$.

Es posible que las diferencias observadas entre especies, en términos de emergencia de plántulas y cobertura, sean un reflejo de las diferentes densidades de siembra usadas en este ensayo ( $6 \mathrm{~kg} \mathrm{ha}^{-1}$ para Mulato y 3 $\mathrm{kg} \mathrm{ha}^{-1}$ para $B$. brizantha), las cuales son las recomendadas por la literatura de lanzamiento, particularmente para Mulato (Grupo
Papalotla, 2003). Se introdujo la cobertura por plántula (\% cobertura/número de plántulas) como una nueva variable de respuesta en un intento por estandarizar los resultados. La Fig. 2 muestra los resultados de la evolución de esta nueva variable para las diferentes épocas de evaluación y bajo las diferentes combinaciones de fertilización. El resultado del análisis de varianza mostró que, en promedio de niveles de fertilización, la cobertura por plántula de Mulato fue significativamente superior a $B$. brizantha a las $3(\mathrm{p}=0.0014)$ y $6(\mathrm{p}=0.0003)$ semanas; sin embargo, las diferencias relativas fueron definitivamente menores; es decir, $10 \%$ para cobertura por plántula, en comparación a $42 \%$ para cobertura total, en promedio de épocas de evaluación y niveles de fertilización.

Los resultados de este intento de estandarización deben ser interpretados con cautela ya que no se pudo encontrar una correlación consistentemente significativa entre cobertura y número de plántulas para las diferentes evaluaciones. 


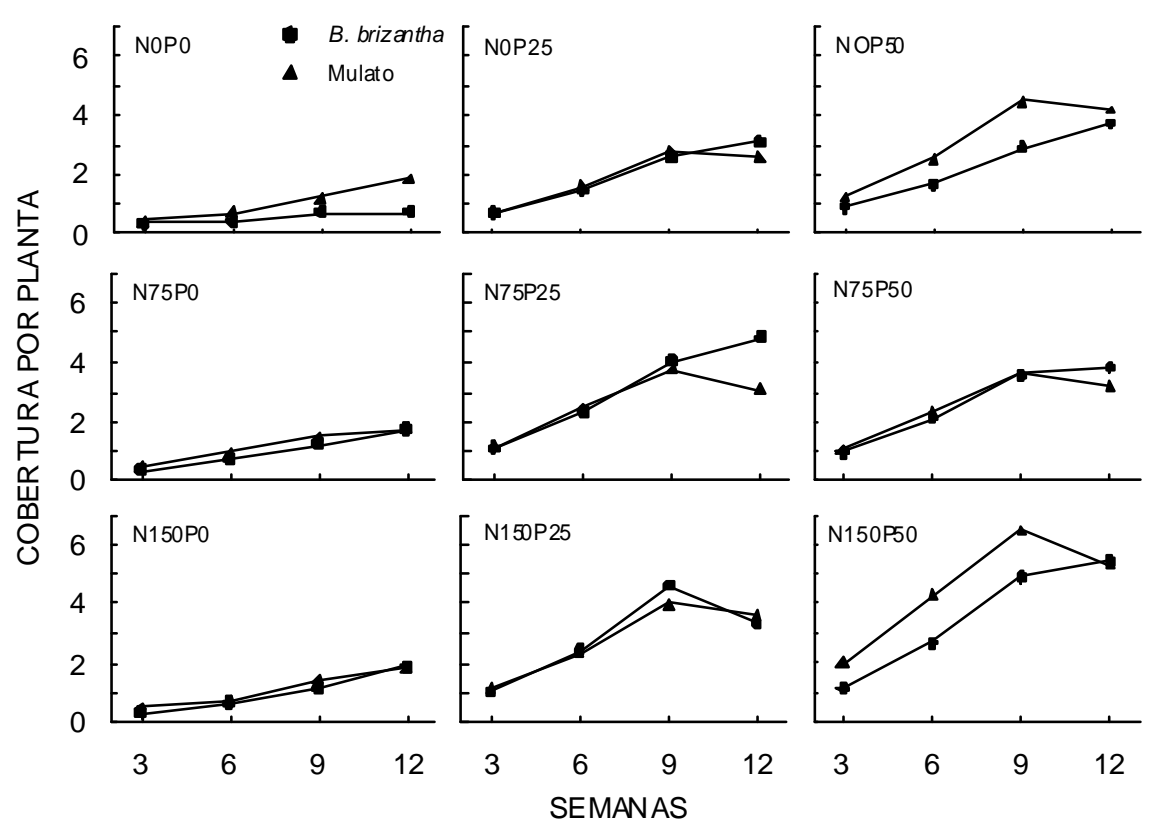

Figura 2. Evolución de la cobertura por plántula de Mulato (Brachiaria ruziziensis $x$ B. Brizantha) y Brachiaria brizantha entre 3 y 12 semanas después de la siembra bajo nueve combinaciones de niveles de fertilización ( $\mathrm{kg} / \mathrm{ha} / \mathrm{año})$ de $\mathrm{N}$ y $\mathrm{P}(\mathrm{n}=3)$. Pucallpa, Perú. 2005.

No existe literatura sobre evaluación cuantitativa del establecimiento de Mulato; sin embargo, los resultados de esta evaluación preliminar permiten concluir que esta gramínea posee una excelente capacidad de establecimiento y particularmente una alta velocidad de cobertura, aun en ausencia de $\mathrm{N}$ pero con fertilización fosforada. Estos resultados confirmarían la información de la literatura de lanzamiento en Colombia (Doral, 2003), la cual describe a Mulato como apropiado para los Llanos Orientales, ecosistema con condiciones de suelo similares a Pucallpa. Se espera que evaluaciones posteriores de crecimiento y producividad respalden la aparente superioridad de Mulato.

\section{Literatura Citada}

\section{Centro Internacional de Agricultu- ra Tropical. 2002. Tropical Forages}

Program 2002. Annual Report. Cali, Colombia: CIAT. p 10-11.

2. Doral Trade. 2003. El pasto mulato crece en Colombia. [Internet] Disponible en: ht tp://www.doraltrade.com/ detalleheme. asp?tipo $=$ noticia $\& \mathrm{Id}=397$

3. Grupo Papalotla. 2003. El pasto mulato.mn. [Internet] Disponible en: http://www.grupopa-palotla.com/ prodmulato_interior.htm

4. PérezJA, García E, Enríquez J, Quero A, Pérez J, Hernández A. 2004. Análisis de crecimiento, área foliar específica y concentración de nitrógeno en hojas de pasto "mulato" (Brachiaria híbrido, cv.). Tec Pec Méx 42: 447-458.

5. Toledo JM, Schultze-Kraft R. 1982. Metodología para la evaluación agronómica de pastos tropicales. En: J.M. Toledo (ed). Manual para la evaluación agronómica de la Red Internacional de Pastos Tropicales. Cali, Colombia: Centro Internacional de Agricultura Tropical. p 91-110. 